Research Article

\title{
Morphological Analysis for Three-Dimensional Chaotic Delay Neural Networks
}

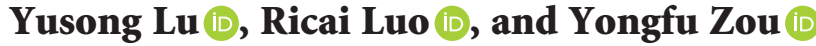 \\ Department of Mathematics and Statistics, Hechi University, Yizhou, China \\ Correspondence should be addressed to Ricai Luo; luoricai@163.com
}

Received 23 August 2020; Revised 19 October 2020; Accepted 26 October 2020; Published 28 November 2020

Academic Editor: Shenggang Li

Copyright (c) 2020 Yusong Lu et al. This is an open access article distributed under the Creative Commons Attribution License, which permits unrestricted use, distribution, and reproduction in any medium, provided the original work is properly cited.

\begin{abstract}
The study focuses on the chaotic behavior of a three-dimensional Hopfield neural network with time delay. We find the aspecific coefficient matrix and the initial value condition of the system and use MATLAB software to draw its graph. The result shows that their shape is very similar to the figure of Rosler's chaotic system. Furthermore, we analyzed the divergence, the eigenvalue of the Jacobian matrix for the equilibrium point, and the Lyapunov exponent of the system. These properties prove that the system does have chaotic behavior. This result not only confirms that there is chaos in the neural networks but also that the chaotic characteristics of the system are very similar to those of Rosler's chaotic system under certain conditions. This discovery provides useful information that can be applied to other aspects of chaotic Hopfield neural networks, such as chaotic synchronization and control.
\end{abstract}

\section{Introduction}

Chaotic systems are nonlinear dynamical systems working in a stochastic process that is neither periodic nor convergent but are highly dependent on the initial value. Particularly, sensitivity to initial conditions implies that any small perturbations in the current trajectory of a dynamic system results in significant differences in future behavior. Chaos exists widely in natural and social fields such as chemistry, physics, mathematics, and biology. The research upsurge for chaos theory began in the early 1970s but the origins of the new discipline can be traced back to the last century. Poincare, a French mathematician and physicist, was the first known scholar to discover chaos. In 1880, Poincare first studied the possibility of chaos [1]. He studied the three-body problem and became the first person to discover the deterministic system of chaos which showed an acyclic behavior dependent on initial conditions. This made long-term prediction impossible, thereby laying the foundation for modern chaos theory. Further contributions by Birkhoff, Cartwright and Littlewood, Levinson, and Kolmogorov, among others, came up later [2].
By 1963, Lorenz, an American meteorologist, discovered a three-dimensional chaotic system which he later called the Lorenz chaotic system while, when studying weather models [3]. The revolutionary work on chaos was discovered from a three-dimensional chaotic system by an American meteorologist, Edward Lorenz, in 1963 while studying weather models. Since then, in-depth studies on chaos theory has been done in science and engineering fields. The theory has important applications in information processing, high performance circuit, secure communication, and other issues $[4-10]$.

Many classical paradigms have emerged since the discovery of Lorenz chaotic systems, such as the famous Rossler chaotic system [11], Lü system [12], Chen system [13], and Cai's circuit chaotic system [14], among others. The Rossler system is the most famous chaotic system having simple asymmetric attractor substructure extracted from the Lorenz attractor by the German physical chemist, Rossler. It plays a vital role in signal processing [15], secure communication [16], and other issues.

In the past decade, the chaos of neural networks has been extensively researched. For example, Yang et al. analyzed the 
transient chaos in a chaos bifurcation problem of a class of simple chaotic Hopfield neural networks. [17]. Zou et al. observed chaotic attractors of nonautonomous cellular neural networks by using antisymbol templates [18], and they found interesting fractal structures. In a study by Das et al., rich dynamic characteristics were revealed based on analysis of artificial neural networks composed of three neurons and they drew bifurcation and three-dimensional phase diagrams of the model [19]. In addition, Zhang et al. presented an example of a two-dimensional chaotic neural network [20]. A proposal by Sampath et al. showed a class of chaotic system with cubic term whereby they analyzed its basic properties [21].

Based on previous ideas and work of Sampath et al., in this paper, we focus on the chaotic behavior of a threedimensional Hopfield neural network with time delay. We presented a neural network model with specific coefficients. To understand the graphical characteristics of this model, we used MATLAB to draw its phase diagram. The result shows that their shape is very similar to the figure of Rosler's chaotic system. Then, we analyzed the divergence, the eigenvalue of the Jacobian matrix for the equilibrium point, and the Lyapunov exponent of the system. These properties prove that the system does have chaotic behavior. This research is a refreshing discovery.

\section{A Three-Dimensional Delay Chaotic Hopfield Neural Network}

We considered the following three-dimensional delay Hopfield neural network:

$$
\dot{\mathbf{x}}(t)=\mathbf{A x}(t)+\mathbf{B} \mathbf{f}(\mathbf{x}(t))+\mathbf{C f}(\mathbf{x}(t-\tau)),
$$

where $\mathbf{x}(t)=\left(x_{1}(t), x_{2}(t), x_{3}(t)\right)$ denotes the state variable, $\mathbf{f}(\mathbf{x}(t))=\left(f\left(x_{1}(t)\right), f\left(x_{2}(t)\right), f\left(x_{3}(t)\right)\right)$ denotes the activation function, and $\tau$ denote the transmission delays and we set $\tau=1, f\left(x_{i}(t)\right)=\tanh x_{i}(t)(i=1,2,3)$, and

$$
\begin{aligned}
& \mathbf{A}=\left(\begin{array}{ccc}
-1 & 0 & 0 \\
0 & -1 & 0 \\
0 & 0 & -1
\end{array}\right), \\
& \mathbf{B}=\left(\begin{array}{ccc}
0 & b_{12} & 0 \\
0 & 0 & b_{23} \\
b_{31} & 0 & b_{33}
\end{array}\right), \\
& \mathbf{C}=\left(\begin{array}{lll}
0 & 0 & 0 \\
0 & 0 & 0 \\
c_{31} & 0 & c_{33}
\end{array}\right) .
\end{aligned}
$$

When we take the following parameter values, system (1) presents a Lossler chaotic attractor:

$$
\begin{aligned}
& b_{12}=30, \\
& b_{23}=90, \\
& b_{31}=0.01, \\
& b_{33}=\sqrt{3}, \\
& c_{31}=0.04, \\
& c_{33}=-1.23,
\end{aligned}
$$

with initial conditions being

$$
\begin{aligned}
& x_{1}(0)=30, \\
& x_{2}(0)=11, \\
& x_{3}(0)=0.12 .
\end{aligned}
$$

Figure 1 displays the three-dimensional view of chaotic neural network system (1) when MATLAB for numerical simulation was used, having one attractor, while Figures 2-4 show the two-dimensional view (projection) of the system's three-dimensional view attractor on three coordinate planes. Figure 5 shows the system (1) state variable $\left(x_{1}(t), x_{2}(t), x_{3}(t)\right)$, respectively, against time $t$.

\section{Properties of Chaotic Systems in Three- Dimensional Delayed Neural Networks}

In this section, we analyzed the basic properties of chaotic systems (1), such as dissipativity, the stability of the equilibrium point, the Lyapunov exponent, and the Kaplan-Yorke dimension.

3.1. Dissipativity. We present the system in another vector form as follows:

$$
\dot{\mathbf{x}}=\mathbf{f}(\mathbf{x})=\left(\begin{array}{l}
f_{1}\left(x_{1}, x_{2}, x_{3}\right) \\
f_{2}\left(x_{1}, x_{2}, x_{3}\right) \\
f_{3}\left(x_{1}, x_{2}, x_{3}\right)
\end{array}\right),
$$

where

$$
\begin{aligned}
& f_{1}\left(x_{1}, x_{2}, x_{3}\right)=-x_{1}+b_{12} * \tanh x_{2}, \\
& f_{2}\left(x_{1}, x_{2}, x_{3}\right)=-x_{2}+b_{23} * \tanh x_{3}, \\
& f_{3}\left(x_{1}, x_{2}, x_{3}\right)=-x_{3}+b_{31} * \tanh x_{1}+b_{33} * \tanh x_{3}+c_{31} * \tanh x_{1}(t-\tau)+c_{33} * \tanh x_{3}(t-\tau) .
\end{aligned}
$$




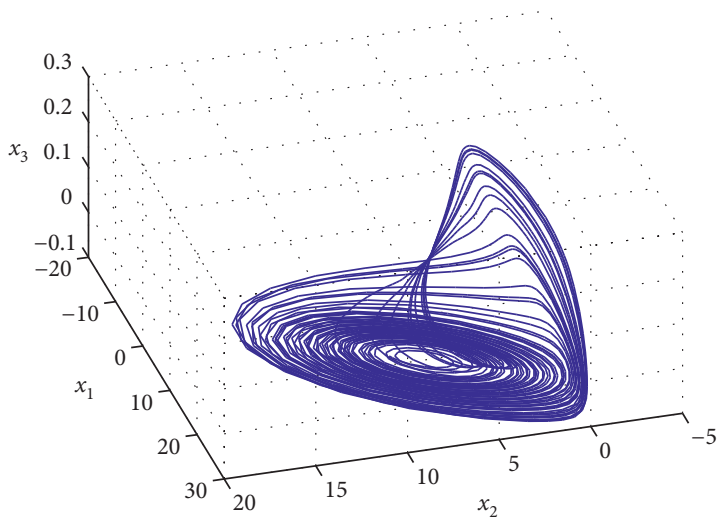

FIgURE 1: Attractor of chaotic neural network system (1).

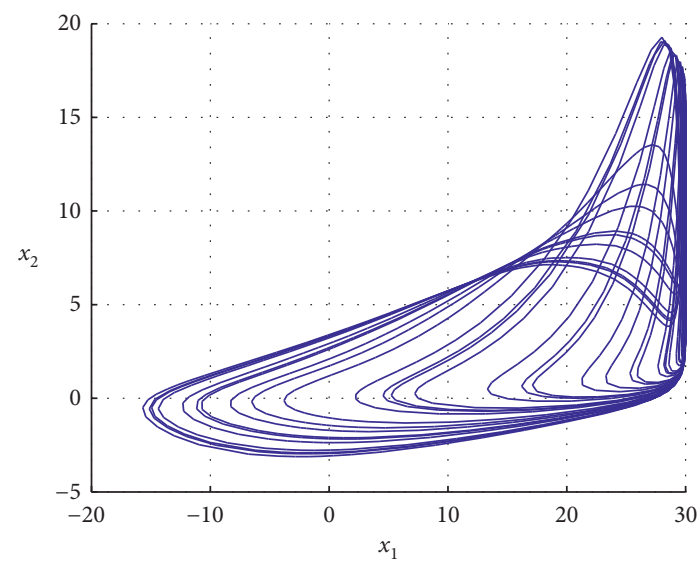

FIgURE 2: Two-dimensional projection of the chaotic neural network system on $x_{1} x_{2}$-coordinate plane.

The parameter value in the chaotic case was named as

$$
\begin{aligned}
& b_{12}=30, \\
& b_{23}=90, \\
& b_{31}=0.01, \\
& b_{33}=\sqrt{3}, \\
& c_{31}=0.04, \\
& c_{33}=-1.23 .
\end{aligned}
$$

Let $\forall \Omega \subset R^{3}$ with smooth boundary, and let $V(t)$ represent the volume of $\Omega(t)$, and according to Liouville's theorem, we obtain

$$
\frac{\mathrm{d} V}{\mathrm{~d} t}=\int_{\Omega(t)}(\nabla \cdot \mathbf{f}) \mathrm{d} x_{1} \mathrm{~d} x_{2} \mathrm{~d} x_{3} .
$$

It is easy to know what the divergence of system (1) is as follows:

$$
\begin{aligned}
\nabla \cdot \mathbf{f} & =\frac{\partial f_{1}}{\partial x_{1}}+\frac{\partial f_{2}}{\partial x_{2}}+\frac{\partial f_{3}}{\partial x_{3}}=-3+b_{33}\left(1-f\left(x_{3}\right)^{2}\right) \\
& =-3+\sqrt{3}\left(1-\left(\tanh x_{3}\right)^{2}\right) \leq-3+\sqrt{3}<0
\end{aligned}
$$

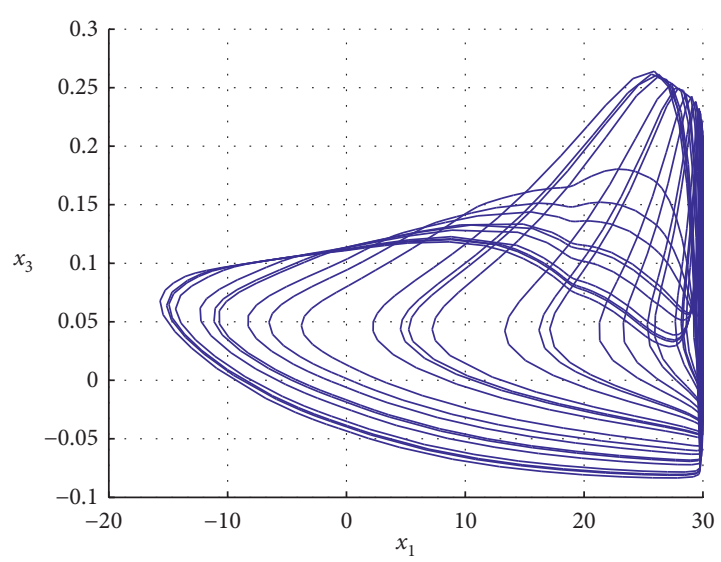

FIgure 3: Two-dimensional projection of the chaotic neural network system on $x_{1} x_{3}$-coordinate plane.

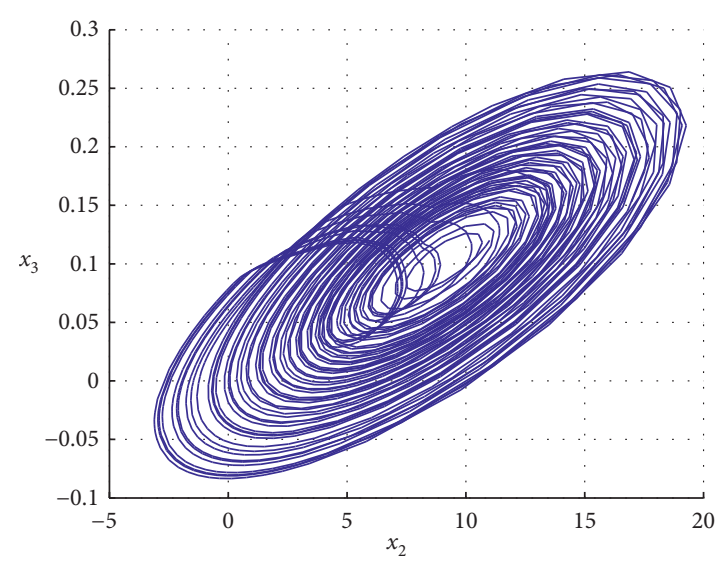

Figure 4: Two-dimensional projection of the chaotic neural network system on $x_{2} x_{3}$-coordinate plane.

Substituting (9) into (8), we obtain

$$
\begin{aligned}
\frac{\mathrm{d} V(t)}{\mathrm{d} t} & =\int_{\Omega(t)}(\nabla \cdot \mathbf{f}) \mathrm{d} x_{1} \mathrm{~d} x_{2} \mathrm{~d} x_{3} \leq \int_{\Omega(t)}(-3+\sqrt{3}) \mathrm{d} x_{1} \mathrm{~d} x_{2} \mathrm{~d} x_{3} \\
& =(-3+\sqrt{3}) V(t) .
\end{aligned}
$$

Since $V(t)>0$, then

$$
\frac{1}{V(t)} \frac{\mathrm{d} V(t)}{\mathrm{d} t} \leq(-3+\sqrt{3})
$$

Integrating both sides of inequality (11) from 0 to $t$, we obtain

$$
V(t) \leq V(0) e^{-(3-\sqrt{3}) t}
$$

It is easy to identify from equation (12) that $\lim _{t \rightarrow 0} V(t)=0$. This result indicated that the system (1) is dissipative.

Thus, the limit set of the system is ultimately limited to a specific limit set of zero volume, and the asymptotic motion of chaotic system (1) is adsorbed to a strange attractor of the system. 


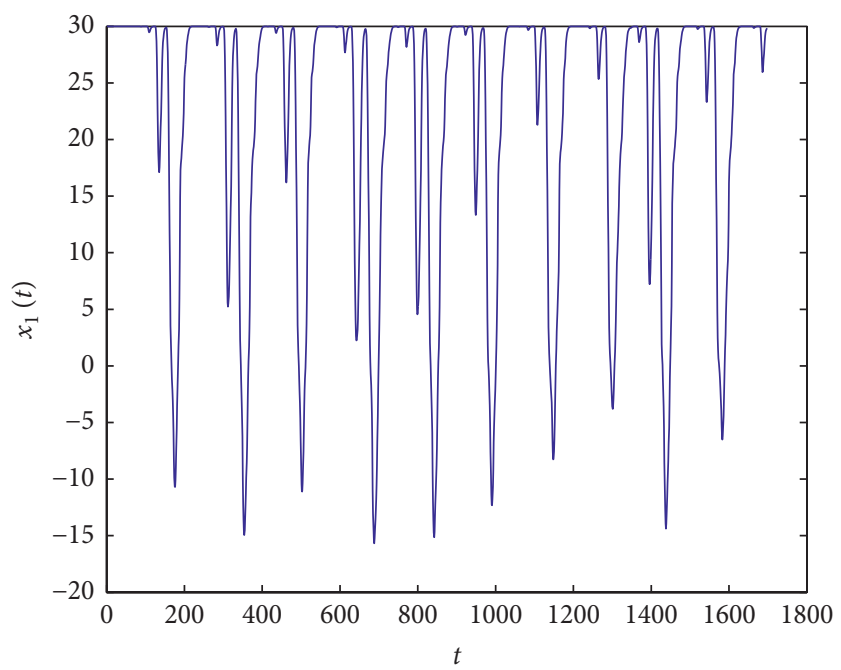

(a)

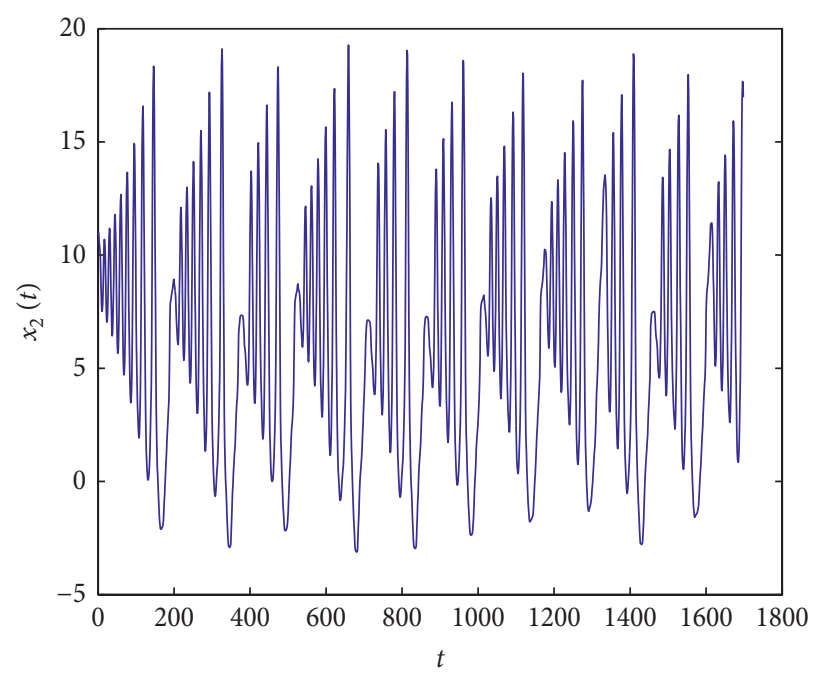

(b)

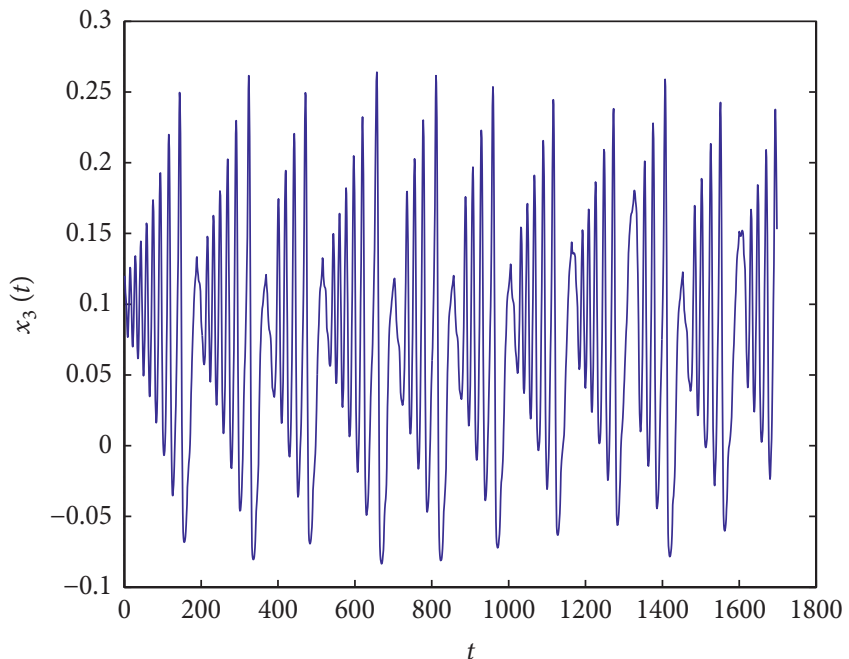

(c)

FIGURE 5: Trajectory components of system (1) $\left(x_{1}(t), x_{2}(t), x_{3}(t)\right)$, respectively, against time for the parameters $b_{12}=30, b_{23}=90$, $b_{31}=0.01, b_{33}=\sqrt{3}, c_{31}=0.04$, and $c_{33}=-1.23$ when the initial state $\left(x_{1}(0), x_{2}(0), x_{3}(0)\right)=(30,11,0.12)$. 
3.2. Stability of the Equilibrium Point. By solving the following equations (where $b_{12}=30 ; b_{23}=90 ; \quad b_{31}=0.01$; $\left.b_{33}=\sqrt{3} ; c_{31}=0.04 ; c_{33}=-1.23\right)$, chaotic system (1) has one equilibrium point, that is, the origin $(0,0,0)$ :

$$
\begin{aligned}
&-x_{1}^{*}+b_{12} * \tanh x_{2}^{*}=0, \\
&-x_{2}^{*}+b_{23} * \tanh x_{3}^{*}=0, \\
&-x_{3}^{*}+\left(b_{31}+c_{31}\right) * \tanh x_{1}^{*}+\left(b_{33}+c_{33}\right) * \tanh x_{3}^{*}=0 .
\end{aligned}
$$

From the above equation, we obtain the Jacobian matrix of system (1):

$$
\left[\begin{array}{ccc}
-1 & b_{12} *\left(1-\tanh ^{2} x_{2}\right) & 0 \\
0 & -1 & b_{23} *\left(1-\tanh ^{2} x_{3}\right) \\
\left(b_{31}+c_{31}\right) *\left(1-\tanh ^{2} x_{1}\right) & 0 & -1+\left(b_{33}+c_{33}\right) *\left(1-\tanh ^{2} x_{3}\right)
\end{array}\right] .
$$

Therefore, we can obtain the Jacobian matrix of system (1) at the equilibrium point as follows:

$$
J=\left[\begin{array}{ccc}
-1 & 30 & 0 \\
0 & -1 & 90 \\
0.05 & 0 & -1+(\sqrt{3}-1.23)
\end{array}\right]
$$

Calculating the eigenvalue of the matrix $J$, we have

$$
\begin{aligned}
\lambda_{1} & =4.3028, \\
\lambda_{2,3} & =-3.4004 \pm 4.4380 i .
\end{aligned}
$$

The equilibrium point was considered a saddle point focus. It can be seen that the equilibrium point of system (1) is unstable.

\subsection{Lyapunov Exponents and Kaplan-Yorke Dimension.}

The Lyapunov exponent is an important parameter to measure a chaotic system. It is usually used to describe the characteristics of the motion of a system. Its positive values and negative values along a certain direction indicate the average divergence or convergence speed of the adjacent orbitals in the attractor for a long time. When Lyapunov exponent is less than 0 , it indicates that the phase volume will shrink, the motion state of the system will tend to be stable, and the system is not sensitive to the initial state. When the Lyapunov exponent is greater than 0 , it indicates that the phase volume will expand and the adjacent two orbitals will gradually separate and have more and more differences, so that the motion state of the system will finally enter into a chaotic state. When Lyapunov exponent is equal to 0 , the system is in critical stable situation. If the system is in a chaotic state, there must be a Lyapunov exponent greater than zero. Therefore, judging the magnitude, positive and negative of the Lyapunov exponent become a criterion for whether the system enters chaos [22-25].

To analyze the chaotic behavior of system (1) by using the Lyapunov exponents, for parameter value (3) and initial condition (4), by using MATLAB, we obtained the Lyapunov exponents of the system (1) as follows:

$$
\begin{aligned}
& \lambda_{1}=0.15, \\
& \lambda_{2}=0, \\
& \lambda_{3}=-9.009 .
\end{aligned}
$$

From this, system (1) has three Lyapunov exponents: positive, negative, and zero. The maximum Lyapunov exponent of system (1) is $\lambda_{1}=0.15$. This makes the initial conditions unpredictable to the long-term behavior of the system. System (1) should therefore present a chaotic phenomenon which is consistent with the numerical simulation.

The Kaplan-Yorke dimension (KYD) was obtained using the Lyapunov index of system (1) as in the following equation:

$$
D_{\mathrm{KY}}=k+\frac{\sum_{i}^{k} \lambda_{i}}{\left|\lambda_{k+1}\right|},
$$

where $k$ is an integer and $k+1$ is the number of Lyapunov exponents. The number of Lyapunov exponents of system (1) is equal to the number of state variables, that is, 3 . When equation (1) presents chaotic behavior, $k=2$ and $\lambda_{k+1}=\lambda_{3}$ is the third Lyapunov exponent (in descending order). Therefore, from equation (18), the KYD of system (1) is

$$
D_{\mathrm{KY}}=2+\frac{\lambda_{1}+\lambda_{2}}{\left|\lambda_{3}\right|}=2.01665 \text {. }
$$

\section{Conclusion}

In summary, we draw a graph of a neural network model with a specific coefficient matrix and analyze the properties of the system, such as equilibrium point, divergence, and Lyapunov exponent. This result proves that the neural network not only has chaos but also has similar chaotic characteristics with Rosler's chaotic system under some 
specific parameters, although their equations are different. This discovery provides useful information that can be applied to other aspects of chaotic Hopfield neural networks, such as chaotic synchronization and control.

\section{Data Availability}

The data used to support the findings of this study are included within the article.

\section{Conflicts of Interest}

The authors declare that they have no conflicts of interest.

\section{Acknowledgments}

This work was supported by the National Science Foundation of China (11961021 and 11561019), Guangxi Natural Science Foundation (2020GXNSFAA159084), Hechi University Research Fund for Advanced Talents (2019GCC005), and Scientific Research Foundation of the Education Department of Guangxi Autonomous Region of China (2019KY0625).

\section{References}

[1] J. H. Poincaré, "Sur le problème des trois corps et les équations de la dynamique, Divergence des séries de M. Lindstedt," Acta Mathematica, vol. 13, pp. 1-270, 1890.

[2] J. Hadamard, "Les surfaces à courbures opposées et leurs lignes géodesiques," Journal de Mathématiques Pures et Appliquées, vol. 4, pp. 27-73, 1898.

[3] E. N. Lorenz, "Deterministic nonperiodic flow," Journal of the Atmospheric Sciences, vol. 20, no. 2, pp. 130-141, 1963.

[4] Y. V. Andreyev, A. S. Dmitriev, E. V. Efremova, and A. N. Anagnostopoulos, "Chaotic signal processing: information aspects," Chaos, Solitons \& Fractals, vol. 17, no. 2-3, pp. 531-544, 2003.

[5] F. Zhu, "Observer-based synchronization of uncertain chaotic system and its application to secure communications," Chaos, Solitons \& Fractals, vol. 40, no. 5, pp. 2384-2391, 2009.

[6] J. Feng, C. K. Tse, and F. C. M. Lau, "Reconstruction of chaotic signals with application to channel equalization in chaosbased communication systems," International Journal of Communication Systems, vol. 17, no. 3, pp. 217-232, 2004.

[7] M. Morisue, M. Yamadaya, H. Noguchi, and A. Kanasugi, "A digital application of chaotic oscillation modes in Josephson circuit," International Journal of Intelligent Systems, vol. 12, no. 4, pp. 267-290, 1997.

[8] M. E. Sahin, Z. G. Cam Taskiran, H. Guler, and S. E. Hamamci, "Simulation and implementation of memristive chaotic system and its application for communication systems," Sensors and Actuators A: Physical, vol. 290, pp. 107-118, 2019.

[9] X. Li, X. Yang, and T. Huang, "Persistence of delayed cooperative models: impulsive control method," Applied Mathematics and Computation, vol. 342, pp. 130-146, 2019.

[10] D. Yang, X. Li, and J. Qiu, "Output tracking control of delayed switched systems via state-dependent switching and dynamic output feedback," Nonlinear Analysis: Hybrid Systems, vol. 32, pp. 294-305, 2019.

[11] O. E. Rössler, "An equation for continuous chaos," Physics Letters A, vol. 57, no. 5, pp. 397-398, 1976.
[12] J. Lü, G. Chen, and D. Cheng, "A new chaotic system and beyond: the generalized Lorenz-like system," International Journal of Bifurcation and Chaos, vol. 14, no. 5, pp. 1507-1537, 2004.

[13] G. Chen and T. Ueta, "Yet another chaotic attractor," International Journal of Bifurcation and Chaos, vol. 9, no. 7, pp. 1465-1466, 1999.

[14] G. Cai and Z. Tan, "Chaos synchronization of a new chaotic system via nonlinear control," Journal of Uncertain Systems, vol. 1, pp. 235-240, 2007.

[15] D. Cafagna and G. Grassi, "Observer-based projective synchronization of fractional systems via a scalar signal: application to hyperchaotic Rössler systems," Nonlinear Dynamics, vol. 68, no. 1-2, pp. 117-128, 2012.

[16] C.-K. Chen, J.-J. Yan, and T.-L. Liao, "Sliding mode control for synchronization of Rössler systems with time delays and its application to secure communication," Physica Scripta, vol. 76, no. 5, pp. 436-441, 2007.

[17] X.-S. Yang and Q. Yuan, "Chaos and transient chaos in simple Hopfield neural networks," Neurocomputing, vol. 69, no. 1-3, pp. 232-241, 2005.

[18] F. Zou and J. A. Nossek, "A chaotic attractor with cellular neural networks," IEEE Transactions on Circuits and Systems, vol. 38, no. 7, pp. 811-812, 1991.

[19] A. Das, A. B. Roy, and P. Das, "Chaos in a three dimensional neural network," Applied Mathematical Modelling, vol. 24, no. 7, pp. 511-522, 2000.

[20] H. Zhang and Y. Xie, "Adaptive synchronization between two different chaotic neural networks with time delay," IEEE Transactions on Neural Networks, vol. 18, no. 6, pp. 1841-1845, 2007.

[21] S. Sampath, S. Vaidyanathan, C. K. Volos, and V. T. Pham, "An eight-term novel four-scroll chaotic system with cubic nonlinearity and its circuit simulation," Journal of Engineering Science and Technology Review, vol. 8, no. 2, pp. 1-6, 2015.

[22] A. Silva-Juarez and G. Rodriguez-Gomez, "Optimizing the Kaplan-Yorke dimension of chaotic oscillators applying DE and PSO," Technologies, vol. 7, no. 38, pp. 1-10, 2019.

[23] H. Liu, Y. Pan, S. Li, and Y. Chen, "Synchronization for fractional-order neural networks with full/under-actuation using fractional-order sliding mode control," International Journal of Machine Learning and Cybernetics, vol. 9, no. 7, pp. 1219-1232, 2018.

[24] H. Liu, Y. Pan, J. Cao, H. Wang, and Y. Zhou, "Adaptive neural network backstepping control of fractional-order nonlinear systems with actuator faults," IEEE Transactions on Neural Networks and Learning Systems, vol. 1, 2020.

[25] M. Cencini and F. Cencini, Chaos from Simple Models to Complex Systems, World Scientific, PA, USA, 2010. 